\title{
A Parametric Estimation Approach to for Effective Cluster Selection in WPAN
}

\author{
Kiran \\ Research Scolar, MTech \\ Deptt. Of CSE, \\ HCTM Technical Campus \\ Kaithal, India
}

\author{
Anshu Parashar \\ Associate Professor, \\ Deptt. Of CSE, \\ HCTM Technical Campus \\ Kaithal, India
}

\begin{abstract}
WPAN is the most advanced communication network that combines the capabilities of Mobile network and sensor network in a complex global area scenario. This network defines various integrated processes under communication scenario specification. Cluster Selection process is the most common requirement of any WPAN scenario because of the mobility feature for vehicle nodes. In this work, an effective soft-Cluster Selection mechanism is defined under positional and communication parameter analysis. The work is defined for global highway scenario. The obtained results from system show the effective communication rate and lesser communication loss.
\end{abstract}

\section{Keywords}

WPAN, Cluster Selection, Positional Analysis, Highway Scenario

\section{INTRODUCTION}

WPAN is defined as an effective energy communication network defined under integrated communication capabilities with wireless vehicle node communication. In this network system, an intelligent vehicle communication is defined under safety, comfort and optimized and directed path identification. This communication network required he effective route optimization and the effective communication generation so that the minimum communication throughput is obtained [1][2][3]. This kind of network is defined with some specific protocol definition such as GPSR, DSDV etc.

WPAN network is different from mobile network with highly dynamic nature and frequently change in node position and the node exclusion and inclusion in the network. Generally no node is permanent in this network scenario. The nodes enter to the scenario for a fixed interval and then moves to other network. Each network scenario is controlled by a base station [4][5]. As a vehicle switch between the base station networks, this process is considered as Cluster Selection process.

\subsection{Cluster Selection Mechanism}

In vehicular area network as the communication is performed on mobile node and the node moves outside its current coverage range controlled by the base station or the cluster head or road side unit. Then, outside the current coverage range, there can exist multiple such controllers that can take the charge of the node. All the base station or controller devices that find a new node in their coverage range send the access request to that node. This process of request generation is considered as request poll [6][7][8]. As a vehicle node get the poll request from multiple base station, it has to elect a base station that will bet the node control.

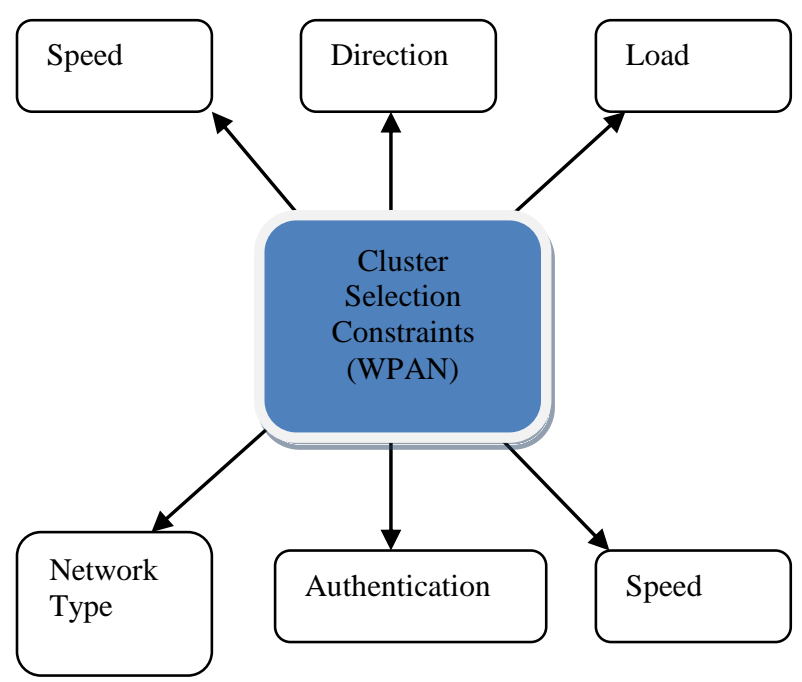

Figure 1: Cluster Selection Constraints

The identification of most effective base station for a node is done using the Cluster Selection mechanism analysis. This analysis is done under the base station strength analysis. From this analysis, the effective base station identification is done. After this identification, the control of the node shifted from earlier base station to this new base station. This process of switching a node between base stations is called Cluster Selection mechanism [9][10]. The Cluster Selection mechanism in vehicular area network is having number of challenges shown in figure 1

WPAN is most dynamic communication network in which nodes enter to the system for a small interval and the speed of the nodes is very high because of this there is the requirement of a fast analytical approach that perform the analysis on multiple parameters. These parameters include the positional parameters such as speed and direction. A predictive analysis approach is required to analyze the position or localization of nodes so that effective Cluster Selection will be obtained. The direction aspect is quite important, because if two base stations are having node coverage then the base station, in direction of which node is moving is more effective. Because over the distance, the signal will become stronger. The base station load is the parameter to decide the network capability [11][12]. A base station with heavy traffic cannot provide effective Cluster Selection.

In WPAN, the Cluster Selection can be controlled by different kind of network devices such as a cluster head, base station or the RSU. The decision of the responsible component is based on the type of communication scenario. A network type with group mobility performs the Cluster Selection over the cluster whereas in city scenario like network base station can be considered. Another factor reliable Cluster Selection process is the authenticated communication. If the key sharing 
authentication is defined in such case before the Cluster Selection process, authentication is required to apply [13].

According to the type of networks involved in the Cluster Selection mechanism, the Cluster Selection is divided in two main classes called horizontal Cluster Selection and vertical Cluster Selection. If the Cluster Selection is performed between two similar networks it is called Horizontal Cluster Selection but if the Cluster Selection is performed between two dissimilar networks, the Cluster Selection mechanism is called vertical Cluster Selection. This mechanism is called network switching mechanism. The hybrid networks enable the vertical Cluster Selection. The classification of Cluster Selection process depends on the base stations and the associated applications. This kind of Cluster Selection mechanism is called break before make Cluster Selection mechanism. It enables the one to one communication under the connection network analysis. This network type defines the break connection while performing the Cluster Selection process. The soft Cluster Selection whereas first make the connection with new one and then perform the break with previous one [14]. This does not give any data loss during Cluster Selection process.

In this paper, an effective Cluster Selection mechanism is defined for vehicular area network. The approach has used the communication and positional parameters for effective base station identification. In this section, an introduction to vehicular area network and Cluster Selection mechanism is defined. In section II, the work defined by earlier researchers is discussed. In section III, the proposed WPAN communication architecture is presented. In section IV, the results obtained from the work are discussed. In section IV, the results obtained from the work are presented. In section V, conclusion obtained from the work is presented.

\section{EXISTING WORK}

In this section, the work done by the earlier researchers on different issues and challenges associated with vehicular network is discussed. This section has defined the contribution of the earlier researchers. B. Karp and H.T. Kung [1] have defined a GPSR based routing approach to provide effective communication in WPAN. Author has defined the routing protocol specification under complexity analysis and packet delivery analysis in a dense communication network. Author defined the complexity analysis and packet delivery analysis in traffic analysis in route length optimization for protocol message generation and mobility analysis so that the data packet transmission is not performed. Data packet is defined under protocol specification. Johnson and Maltz[2] have defined a traffic analysis approach using DSR. Author defined the packet throughput in the network. Author defined the route definition and request analysis so that the destination analysis for propagation for request analysis.

C.Lochert [4] has defined an improved communication for position based routing and Cluster Selection process. Author defined work for city scenario and provides the effective network communication in such network. Author defined the traffic analysis under multiple parameters and provided topology specific communication to improve delivery rate and latency. Author [7] also defined the GPSR based routing to improve the communication throughput. Author presented the comparative study with AODV and DSR protocols so that effective delivery rate will be obtained and the delay will be reduced over the communication. H. FulBler [8] has presented a location analysis based approach for optimized Cluster
Selection process for highway scenario. Author defined the topology based mechanism to provide effective route generation in city scenario. Author presented the broader view under route optimization. Author defined multihop communication approach for real word scenario.

Author [7] refers to vehicles that link WPAN with the 3G/UMTS network. The present paper addresses these concerns in the envisioned WPAN-UMTS integrated architecture and delineates the methodology of dynamic clustering and adaptive gateway management. Author [9] explores geographical location awareness to support software agent mobility in ad hoc networks. The idea is to evaluate the concept of opportunistic communication to perform agent migration and mobility among nodes (Cluster Selection), in an infrastructure less vehicular ad-hoc network (WPAN). Author[10] analyzed several Ad hoc routing communications protocol, Responsive, Proactive \& Hybrid, lecturing in to consideration several Vehicular Ad-Hoc Network argument corresponding Speed, height etc in actual traffic Scenario. The parameters of Vehicular Ad-Hoc Network (WPAN) are changing demonstrates that in the actual traffic scenarios proactive communication protocol accomplish more efficiently toward energy preservation [8]. Author [11] studied various Ad hoc routing protocols, Reactive, Proactive \& Hybrid, taking in to consideration various WPAN parameters like speed, altitude etc in real traffic scenario and evaluated them for various battery models for energy conservation. Author [12] defined novel routing protocols for a sparse environment in WPAN with the context of utilizing the mobility feature, with the aid of the equipped devices, such as Global Position System (GPS) and Navigation System (NS). This approach exploits the knowledge of Second Heading Direction (SHD), which represents the knowledge of the next road direction the vehicle is intending to take, in order to increase the packet delivery ratio, and to increase the route stability by decreasing instances of route breakage.

\section{PROPOSED MODEL}

When we work in a personal area network, in such network the smart sensor devices are attached with different products that can be in static or in moving state. Such as the PAN area defined in a hospital can have some moving devices. Each sensing device is controlled by some controller device. Each controller device is defined with some range specification in which it can control the sensing devices. When these moving sensor moves outside the sensing area then it is required by some other controller device to take the charge of this sensing device. The process of taking the charge or control of some device by some controller is called handoff process. In PAN area where accuracy or the effective throughput is one of the critical factor handoff processes is required to be very effective. In this present work, an effective parametric handoff process is proposed in case of wireless PAN.

The presented work is parametric defined under 4 major parameters called energy, direction, throughput and the capacity. As the handoff process is performed, a link break is performed as the node is outside its current sensing range. Now the entire controller device set their eligibility to the node to take its control. This process is called polling. Now as the polling performed, the analysis will be performed to identify the most eligible controller for the device. At the first stage, the physical characteristics of the controller devices are taken such as the capacity and the throughput. Base on these analyses the primary decision is taken. Later on at the second level, the direction based analysis is performed. To take this analysis the throughput and distance is analyzed in two time 
frames. If the distance towards a node is decreasing it shows that the node is moving to that node's direction and the handoff will be performed to that node only. As the network is a sensor network with energy limit, the energy estimation in the handover process is also required to estimate. Here energy is taken as the secondary parameter.

\begin{tabular}{|c|c|}
\hline \multicolumn{2}{|c|}{ Algorithm () } \\
\hline 1. & $\begin{array}{l}\text { Define a network with } \mathrm{N} \text { Nodes with clustered } \\
\text { Topology }\end{array}$ \\
\hline 2. & $\begin{array}{l}\text { Define the Clusters over the network with effective } \\
\text { controller node }\end{array}$ \\
\hline 3. & $\begin{array}{l}\text { For } \mathrm{i}=1 \text { to Count (Clusters) } \\
\text { [Process All Clusters] } \\
\{ \\
\text { For } \mathrm{j}=1 \text { to } \mathrm{N} \\
\{\end{array}$ \\
\hline 4. & $\begin{array}{l}\text { if (Distance(Node(j),Cluster(i)) } \\
<\text { SensingRange) } \\
\{\end{array}$ \\
\hline 5. & $\begin{array}{l}\text { Cluster(i).Add }(\operatorname{Node}(\mathrm{j})) \\
\} \\
\}\end{array}$ \\
\hline 6. & $\begin{array}{l}\text { Select the Random Set of Pairs of Nodes for Inter } \\
\text { and Intra-Cluster Communication }\end{array}$ \\
\hline 7. & $\begin{array}{l}\text { For } i=1 \text { to Length(Pairs) } \\
\text { [Process All Communication Pairs] } \\
\{\end{array}$ \\
\hline 8. & $\begin{array}{l}\text { Set } \operatorname{Src}=\text { Pairs }(1) \\
\text { Set Dst=Pairs(2) } \\
\text { [Set source and destination node for each node } \\
\text { pair] }\end{array}$ \\
\hline 9. & $\begin{array}{l}\text { if(CommunicationType }(\mathrm{Src}, \mathrm{Dst})=\text { "Intra- } \\
\text { Cluster") } \\
\{\end{array}$ \\
\hline 10. & $\begin{array}{ll}\text { Perform } & \text { Communication } \\
\text { Between(Src,ClusterHead) } & \end{array}$ \\
\hline & $\begin{array}{l}\text { Perform } \\
\text { Between(ClusterHead,Dst) Communication } \\
\}\end{array}$ \\
\hline 11. & $\begin{array}{l}\text { else } \\
\{\end{array}$ \\
\hline 12. & $\begin{array}{l}\text { Cluster1=IdentifyCluster(Src) } \\
\text { Cluster2=IdentifyCluster(Dst) } \\
\text { PerformCommunication(Src,Cluster1) } \\
\text { PerformCommunication(Cluster1,Cluster2) } \\
\text { PerformCommunication(Cluster2,Dst) } \\
\text { \} } \\
\text { \} }\end{array}$ \\
\hline 13. & $\begin{array}{l}\text { As Node moves outside its cluster range identify } \\
\text { the adaptive cluster list }\end{array}$ \\
\hline 14. & $\begin{array}{l}\text { Identify the cluster with effective throughput, } \\
\text { capacity and idle Rate called cluster C }\end{array}$ \\
\hline $\begin{array}{l}15 . \\
\} \\
\}\end{array}$ & ShiftControl (Node, Cluster C) \\
\hline
\end{tabular}

\section{RESULTS}

In this present work, parametric Cluster Selection mechanism is defined for WPAN network. The work includes the positional and communication parameter analysis. The is implemented in NS2 network. The simulation parameters considered in this work are shown in table 2

\section{Table 2: Simulation Parameters}

\begin{tabular}{|l|l|}
\hline Parameter & Value \\
\hline Frequency Band & $5 \mathrm{MHz}$ OFDM \\
\hline Modulation Scheme & $1 / 2 \mathrm{BPSK}$ \\
\hline No of BS & 5 \\
\hline No of MS & 50 \\
\hline $\begin{array}{l}\text { No of active MS under each } \\
\text { BS }\end{array}$ & 5 \\
\hline Simulation duration & $10 \mathrm{~s}$ \\
\hline Requested data rate & $50 \mathrm{kbps}$ \\
\hline BS coverage & $10 \mathrm{~m}$ \\
\hline Propagation model & Two ray ground \\
\hline Antenna Model & Omni directional \\
\hline MS Speed & $5 \mathrm{~m} / \mathrm{min}$ \\
\hline
\end{tabular}

The analysis of work is defined in terms packet loss analysis and communication delay analysis. The results obtained from the work are shown here

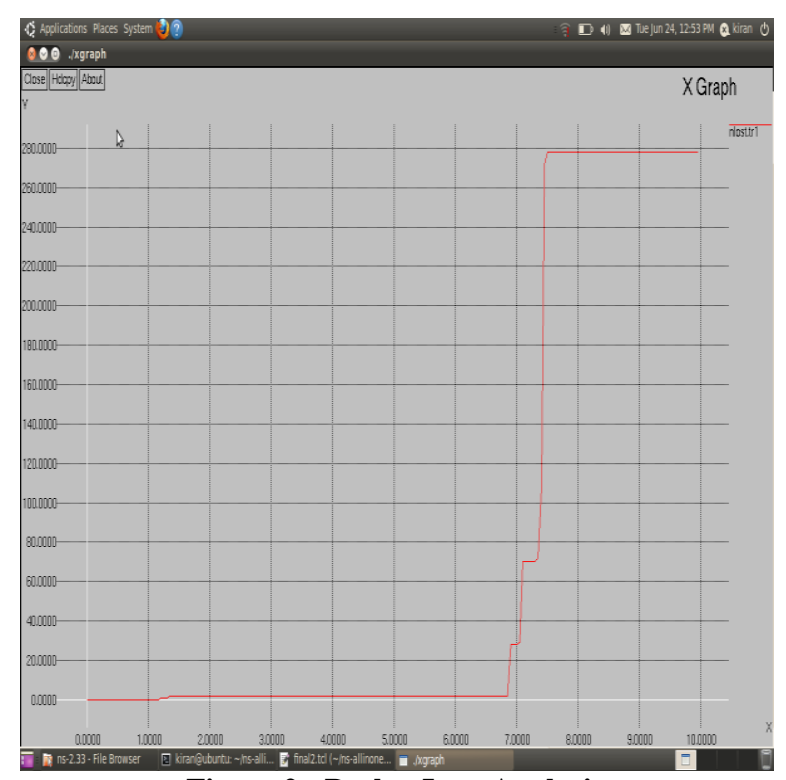

Figure 2 : Packet Loss Analysis

Figure 2 is showing the packet loss analysis in proposed approach and the default adaptive approach of protocol. Here $\mathrm{x}$ axis represents the simulation time and $\mathrm{y}$ axis represents the packet loss. Figure is showing the proposed work has provided the effective communication over the network in specified time. The figure shows that the proposed approach as reduced the packet loss over the network.

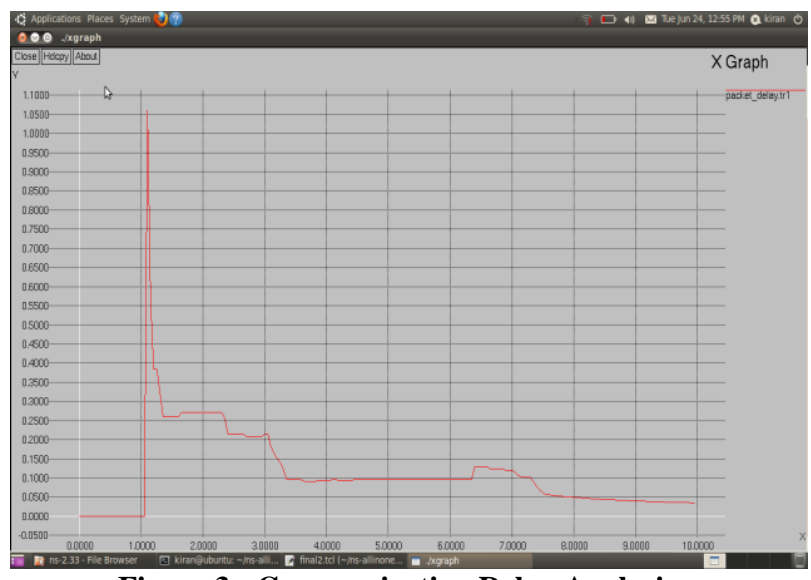

Figure 3 : Communication Delay Analysis 
Figure 3 showing the packet Delay analysis in proposed approach and the default adaptive approach of protocol. Here $\mathrm{x}$ axis represents the simulation time and $\mathrm{y}$ axis represents the packet delay. Figure is showing the proposed work has provided the effective communication over the network in specified time. The figure shows that the proposed approach as reduced the packet delay over the network

\section{CONLCUSION}

In this paper, an effective parametric analysis approach is defined for Cluster Selection process optimization in WPAN network. The network is defined with $\mathrm{N}$ number of cluster formation where each sub network is defined with a base station. All these sub networks are controlled under Zigbee Protocol. As the node moves outside the cluster, the cluster selection process is performed based on positional and communication parameters. The work is here defined to perform the effective cluster election when node moves outside current cluster. The obtained results show that the presented work has improved the packet communication and reduced the communication loss and delay. The work can be improved in future on other network types such as vehicular area network and other network scenarios.

\section{REFERENCES}

[1] B. Karp and H.T. Kung, "GPSR: Greedy perimeter stateless routing for wireless networks," in Proceedings of the ACM/IEEE International Conference on Mobile Computing and Networking (MobiCom), 2000.

[2] D.B. Johnson and D.A. Maltz, "Dynamic source routing in ad hoc wireless networks". In Mobile Computing, T. Imielinski and $\mathrm{H}$. Korth, Eds. Kluwer Academic Publishers, 1996, ch. 5, pp. 153-181.

[3] KARP, B. Geographic routing for wireless networks. Presentation at AFOSR MURI ACTCOMM Research Review Meeting, Oct. 1998.

[4] Lochert, C., Mauve, M., Füßler, H., and Hartenstein, H., "Geographic routing in city scenarios" SIGMOBILE Mob. Comput. Commun. Rev. 9, 1 (Jan. 2005), pp. 69-72.

[5] Karp, B., Geographic Routing for Wireless Networks, $\mathrm{Ph} . \mathrm{D}$. Dissertation, Harvard University, Cambridge, MA, October, 2000.

[6] M. Jerbi, S. M. Senouci, T. Rasheed., "Towards Efficient Geographic Routing in Urban Vehicular Networks,"
Vehicular Technology, IEEE Transactions on, vol. 58, no. 9, 2009, pp. 5048-5059.

[7] C.Lochert, H. Hartenstein, J.Tian, D. Herrmann, H. Fu1Bler, and M. Mauve, "A routing strategy for vehicular ad hoc networks in city environments," in proceedings of IEEE Intelligent Vehicles Symposium (IV2003)

[8] H. Fu1Bler, M. Mauve, H. Hartenstein, M. Kasemann, and D. Vollmer, "Location based routing for vehicular ad-hoc networks," ACM SIGMOBILE Mobile Computing and Communications Review (MC2R), vol. 7, no. 1,Jan. 2003, pp. 47-49.

[9] Tee, C.A.T.H.; Lee, A.C.R., "Survey of position based routing for Inter Vehicle Communication system", Distributed Framework and Applications, 2008. DFmA 2008. First International Conference on, Oct. 2008, pp.174-182, 21-22.

[10] M. Mauve, A. Widmer, and H. Hartenstein, "A survey on position-based routing in mobile ad hoc networks," Network, IEEE, vol. 15, no. 6, Nov/Dec 2001, pp. 30 39.

[11] Takano, A.; Okada, H.; Mase, K., "Performance Comparison of a Position-Based Routing Protocol for WPAN", Mobile Adhoc and Sensor Systems, 2007. MASS 2007. IEEE Internatonal Conference, Oct.2007, pp.1-6.

[12] Liu, B.-S. Lee, B.-C. Seet, C.H. Foh, K.J. Wong, and K.K. Lee, "A routing strategy for metropolis vehicular communications," in International Conference on Information Networking (ICOIN), pp. 134-143, 2004

[13] R.A. Santos, A. Edwards, R. Edwards, and L. Seed, "Performance evaluation of routing protocols in vehicular ad hoc networks," The Intemational Joumal of Ad Hoc and Ubiquitous Computing, vol. 1, no. 1/2, 2005, pp. 80-91.

[14] S.Y. Wang, C.C. Lin, Y.W. Hwang, K.C. Tao, and C.L. Chou, "A practical routing protocol for vehicle-formed mobile ad hoc networks on the roads," in Proceedings of the 8th IEEE International Conference on Intelligent Transportation Systems, 2005, pp. 161-165. 